\title{
Analytic approximations for the elastic moduli of two-phase materials
}

\author{
Zhang, Z. J.; Zhu, Y. K.; Zhang, P.; Zhang, Y. Y.; Pantleon, Wolfgang; Zhang, Z. F.
}

Published in:

Physical Review B

Link to article, DOI:

10.1103/PhysRevB.95.134107

Publication date:

2017

Document Version

Publisher's PDF, also known as Version of record

Link back to DTU Orbit

Citation (APA):

Zhang, Z. J., Zhu, Y. K., Zhang, P., Zhang, Y. Y., Pantleon, W., \& Zhang, Z. F. (2017). Analytic approximations for the elastic moduli of two-phase materials. Physical Review B, 95(13), [134107].

https://doi.org/10.1103/PhysRevB.95.134107

\section{General rights}

Copyright and moral rights for the publications made accessible in the public portal are retained by the authors and/or other copyright owners and it is a condition of accessing publications that users recognise and abide by the legal requirements associated with these rights.

- Users may download and print one copy of any publication from the public portal for the purpose of private study or research.

- You may not further distribute the material or use it for any profit-making activity or commercial gain

- You may freely distribute the URL identifying the publication in the public portal 


\title{
Analytic approximations for the elastic moduli of two-phase materials
}

\author{
Z. J. Zhang, ${ }^{1,}{ }^{*}$ Y. K. Zhu, ${ }^{1}$ P. Zhang, ${ }^{1}$ Y. Y. Zhang, ${ }^{1}$ W. Pantleon, ${ }^{2}$ and Z. F. Zhang ${ }^{1, \dagger}$ \\ ${ }^{1}$ Shenyang National Laboratory for Materials Science, Institute of Metal Research, Chinese Academy of Sciences, \\ Shenyang 110016, People's Republic of China \\ ${ }^{2}$ Section of Materials and Surface Engineering, Department of Mechanical Engineering, \\ Technical University of Denmark, 2800 Kongens Lyngby, Denmark
}

(Received 29 August 2016; revised manuscript received 6 March 2017; published 17 April 2017)

\begin{abstract}
Based on the models of series and parallel connections of the two phases in a composite, analytic approximations are derived for the elastic constants (Young's modulus, shear modulus, and Poisson's ratio) of elastically isotropic two-phase composites containing second phases of various volume fractions, shapes, and regular distributions. Comparison with a plentitude of finite element simulations and numerous previous experimental investigations shows a large consistency between the results and the analytic expressions derived, confirming the adequacy of the present approach. Compared with previous classical models, the present model has several advantages, including its simplicity, accuracy of prediction, and universal applicability.
\end{abstract}

DOI: 10.1103/PhysRevB.95.134107

\section{INTRODUCTION}

Two-phase materials are generally applied due to their beneficial combination of mechanical, physical, and electrical properties compared to their constituent elements [1-3]. In the past, numerous theoretical approaches have been proposed to predict these properties based on the properties of the constituent elements [2]. In particular, the elastic properties of a composite such as Young's modulus, shear modulus, and Poisson's ratio have attracted much attention [2-4]. The two most relevant models for the Young's modulus of a two-phase material are the isostrain model of Voigt (for a parallel connection between the phases) and the isostress model of Reuss (for a series connection between the phases) [2]. The corresponding expressions are

$$
\begin{gathered}
E_{\text {parallel }}=E_{1} f_{1}+E_{2} f_{2}, \\
E_{\text {series }}=\frac{E_{1} E_{2}}{E_{1} f_{2}+E_{2} f_{1}}=\left(\frac{f_{1}}{E_{1}}+\frac{f_{2}}{E_{2}}\right)^{-1},
\end{gathered}
$$

in which $f_{1}$ and $f_{2}$ are the respective volume fractions of the two phases. They constitute strict upper and lower bounds for the Young's modulus of the composite. Both models are based on an arrangement of two phases along one specific direction and the assumption of constant strain or constant stress throughout the composite, which possess strict validity only for ideal cases. Actual composites always contain phases nested in each other or surrounded by each other causing heterogeneous distributions of stresses and strains [5]. Several models and theories have been proposed to estimate the Young's modulus for composites of mutually surrounding phases [6-20]. The utilized methods are mainly based upon derivations from elasticity theory [9-16], considerations on the arrangement of the phases, and the sequence of their connection [17-19] or empirical curve fitting to experimental data [20], which lead to either complicated formulae (e.g., requiring summation of determinants) [10-13], or to improved upper and lower

\footnotetext{
*zjzhang@imr.ac.cn

†zhfzhang@imr.ac.cn.
}

bounds only [14-18], or to equations containing parameters that are hard to be determined [20]. Of all these models, the Hashin and Shtrikman bounds (HS bounds) [14], derived from variational principles of elasticity, are broadly accepted to be the most accurate one in describing experimental data [21]. By considering a particular periodic arrangement of cubic inclusions and different connection sequences, Ravichandran [17] provided a set of upper and lower bounds ( $\mathrm{R}$ bounds), which were shown to be narrower limits, much closer to the experimental data than the HS bounds for many second phase reinforced materials. Nevertheless, derivation of upper and lower limits only is still not satisfying. Recently, finite element methods (FEM) have been used extensively to simulate the elastic properties of composite materials based on $2 \mathrm{D}$ or 3D reconstructions of real microstructures [22-26]. The required computational efforts, however, are quite demanding, including imaging of the real microstructures in the first place, reconstruction of the digital images and finally FEM simulation of the elastic behavior.

Therefore, in the present study, an attempt is undertaken to derive analytical expressions for calculating the elastic properties of isotropic two-phase materials explicitly by considering a simple unit cell representing the microstructure of the composite. Several additional assumptions are made: (1) strain compatibility between the reinforcement and matrix, (2) perfect bonding, and (3) negligible elastic interactions between individual particles [17]. For an accurate description of the elastic behavior of the composite, interaction stresses between inclusions should be accounted for [17]. Nevertheless, these interactions are ignored in the present attempt (as well as in Ref. [17]), in order to provide a simple and handy approach. Despite such a simplification, the expressions derived in this manner provide accurate predictions of the elastic moduli compared to both, FEM and experimental data.

The entire derivation is based on similar connection sequences as used in previous studies for deriving the upper and lower bounds $[17,18]$. The important innovation of the present study is to introduce an integration step in the derivation process so that the number of dismounting and joining steps of the unit cell of the composite is increased from the two or four steps used in previous studies $[17,18]$ to infinity. In this manner, 
specific values of the elastic properties are obtained rather than limiting bounds. The derived values are finally compared with a plentitude of FEM simulations and experimental data as well as several classical models, including the Voigt-Reuss (VR) bounds [2], HS bounds [14], and R bounds [17].

\section{DERIVATION PROCESS}

For simplicity, we first investigate a specific two-phase composite consisting of a cube of a second phase surrounded by a matrix consisting of 26 cubes of the same size, as shown by Fig. 1(a). The elastic moduli $E_{1}$ and $E_{2}$ for the second phase and the matrix, respectively, are assumed to be constant. Different sequences of alternating series and parallel connections can be employed for joining the dismounted individual cubes or entire groups of cubes, each leading to a different approximation for the $E$ modulus of the composite. For example, the cube of the second phase can be connected in parallel with the horizontally surrounded eight matrix cubes first, before connecting the obtained horizontal slab in series with the two slabs of $3 \times 3$ matrix cubes on the top and the bottom, as illustrated by Fig. 1(a). This connection sequence can be encoded as $\mathrm{P} 82$ as it starts with a parallel $(\mathrm{P})$ connection and adds first eight and then two additional elements. Likewise, one can also connect the cube of the second phase cube first in series with the two neighboring matrix cubes on the top and the bottom, and then establish a parallel connection with the eight surrounding matrix columns (of three cubes each), as illustrated by Fig. 1(b). This sequence is encoded as S28. Certainly, one can even join the phases in multiple steps, such as connecting the central cube first in parallel with the two
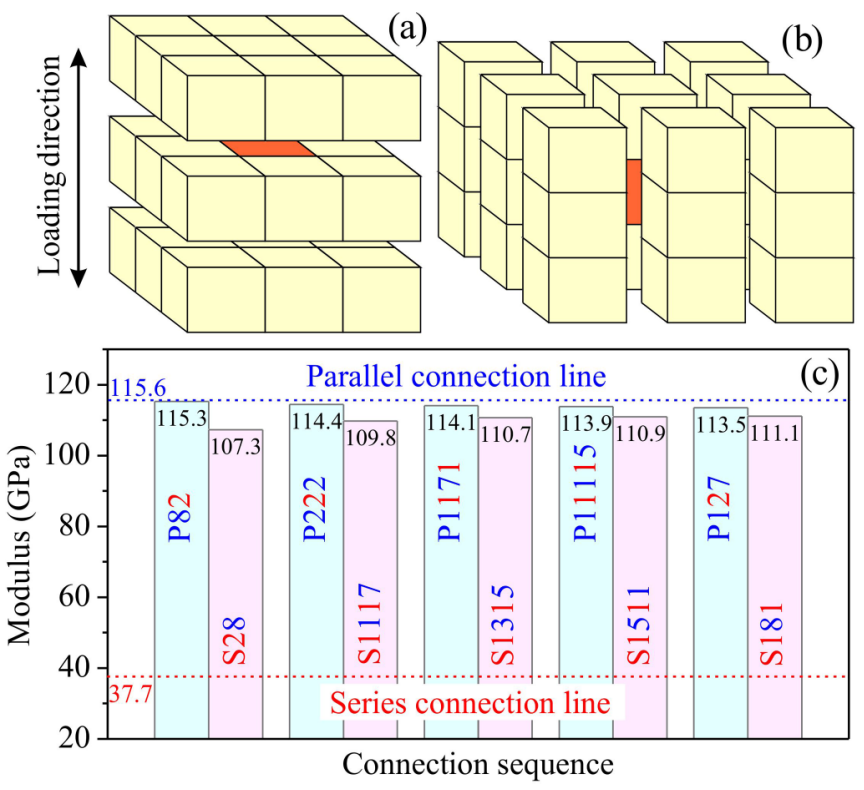

FIG. 1. Illustrations of the various connection sequences [(a) and (b)] for joining the dismounted cubes and the elastic moduli calculated accordingly (c) (with $E_{2}=2 \mathrm{GPa}$ for the inclusion and $E_{1}=120 \mathrm{GPa}$ for the matrix), in which the designations Pxyz/Sxyz represent connection series starting with a parallel or series connection and blue and red numbers mark the number of elements added by parallel and series connection, respectively. matrix cubes in the front and the back, followed by a serial connection with the two matrix rows (consisting of three cubes each) upward and downward, followed in turn by parallel connection with the two matrix slabs of $3 \times 3$ cubes on the right and on the left, encoded as P222, and in many more possible ways. From Eqs. (1) and (2), approximate $E$ moduli for the composite can be calculated for various of such connection sequences.

Figure 1(c) displays the values of the $E$ modulus for all possible connection sequences (for $E_{1}=2 \mathrm{GPa}$ and $E_{2}=$ $120 \mathrm{GPa}$ as an example), in which the upper and lower dotted lines are the values of the Reuss and the Voigt bounds, respectively. The abbreviations Pxyz and Sxyz denote joining operations with an alternating sequence of connecting $x$ elements starting with a parallel or series connection, respectively. Blue and red numbers refer to the number of elements added to the composite in parallel or series connection, respectively. From Fig. 1(c), it becomes obvious that the $E$ moduli of the Pxyz series are always higher than those of the Sxyz series; besides, for any member of the Pxyz (or Sxyz) series holds, that the larger the first number, the higher (or lower, respectively) the resulting $E$ modulus. If the first numbers are equal, the same trend holds for the second number, and so on. It can be concluded that an initial parallel connection yields higher $E$ moduli than an initial series connection and that the first numbers in the designation influence the values more significantly than the later ones, emphasizing the importance of the innermost connection. According to the above rules, the $E$ moduli of S28 and P82 constitute the lowest lower and highest upper bounds among all connection sequences.

These findings on the simple example of 27 equally sized cubes can be generalized: the two two-step sequences, connecting first in parallel then in series (PS-P82 in the special case) and connecting first in series then in parallel (SP-S28 in the special case), constitute the broadest upper and lower limits among all connection sequences. Consequently, the actual value for the $E$ modulus of the composite must be located between these two bounds, as pointed out by previous studies $[17,18]$. For the numerical example above and using only a single set of joining operation, either PS or SP, bounds of 107.3 and 115.3 GPa are obtained and the difference between them becomes $\Delta E=8 \mathrm{GPa}$ [Fig. 1(c)].

Instead of nesting the second phase into the matrix in a single step, the matrix shell can be conceptually divided into a number of $n$ layers like an onion. The composite is then constructed by adding layer (of matrix material) by layer, applying joining operations (PS or SP), while taking into account the proper volume fractions of the nested part. In this manner, even tighter bounds are obtained $\left[(\mathrm{PS})^{n}\right.$ and $(\mathrm{SP})^{n}$ ]: if the matrix shell is divided into 100 thin layers and then joined to a composite layer by layer from inside to outside, the resulting $E$ modulus is calculated by applying either SP or PS joining operations for 100 times $\left[(\mathrm{SP})^{100}\right.$ or (PS $)^{100}$ ] and the resulting bounds are 113.57 and 113.67 $\mathrm{GPa}$ respectively, with a difference of $\Delta E=0.1 \mathrm{GPa}$ in between the two values. If the number of layers is increased further to 10000, the calculated bounds become 113.6135 and $113.6144 \mathrm{GPa}$, respectively, with an even smaller difference $\Delta E=0.0009 \mathrm{GPa}$. 


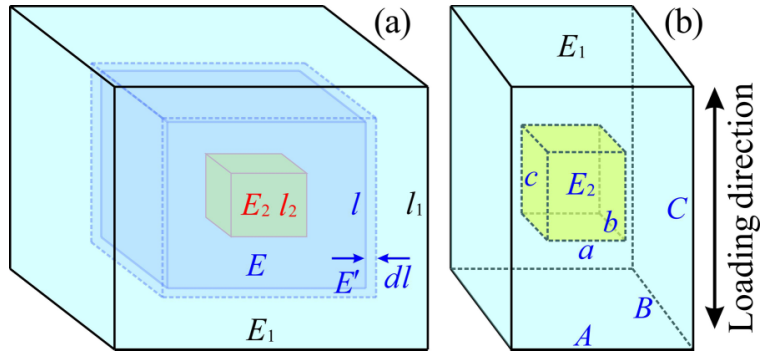

FIG. 2. Schematic representation of the unit cell of the two-phase composites where a cube of a second phase is embedded in a cube of matrix material (a) and a cuboid of a second phase in a cuboid of matrix material (b). The integration method for calculating the elastic moduli of the composite is illustrated in (a) as well.

This Gedanken experiment shows that a continuously decreasing difference $\Delta E$ between the bounds is achieved with increasing number of layers the shell is subdivided into.
This inspires us to presume that if one would increase the subdivision of the matrix to infinity, the difference between the two bounds should become infinitesimal and all the values for the $E$ modulus acquired by various connection sequences should converge into one and the same value, which then must be the real modulus for the given arrangement.

In the following, we will prove the convergence of the two bounds for the $E$ modulus and calculate its value analytically. Figure 2(a) illustrates a composite, in which the moduli and edge lengths of the cubes of the second phase and the matrix are $E_{2}, l_{2}$ and $E_{1}, l_{1}$, respectively. For an inner cube of the two-phase composite with an edge length $l>l_{2}$ [sketched in Fig. 2(a)], the modulus is designated as $E$. When adding a layer of matrix material with infinitesimal thickness $\mathrm{d} l$, the modulus of the composite with edge length $l+d l$ becomes $E^{\prime}=E+d E$. Using Eqs. (1) and (2), the incremental changes $\mathrm{d} E$ calculated according to either an SP or a PS connection sequence become

$$
\begin{gathered}
d E_{S P}=\frac{\left(2 E_{1}^{2}-E^{2}-E E_{1}\right) d t+\left(E_{1}^{2}+E E_{1}-2 E^{2}\right) d t^{2}+\left(E E_{1}-E^{2}\right) d t^{3}}{E_{1}+\left(2 E_{1}+E\right) d t+\left(2 E+E_{1}\right) d t^{2}+E d t^{3}}, \\
d E_{P S}=\frac{\left(2 E_{1}^{2}-E^{2}-E E_{1}\right) d t+\left(3 E_{1}^{2}-3 E E_{1}\right) d t^{2}+\left(E_{1}^{2}-E E_{1}\right) d t^{3}}{E_{1}+\left(2 E_{1}+E\right) d t+3 E_{1} d t^{2}+E_{1} d t^{3}},
\end{gathered}
$$

respectively.

Comparing Eqs. (3) and (4), and omitting high-order terms of $d t=d l / l$ (as $\mathrm{d} t$ is an infinitesimal amount), it is revealed that the coefficients of the leading term are actually identical for both expressions,

$$
d E=\frac{\left(2 E_{1}^{2}-E^{2}-E E_{1}\right)}{E_{1}} \frac{d l}{l},
$$

indicating that after refining the calculations, the values for the $E$ modulus acquired by various connection sequences converge to the same value. In a similar way, the decrease in the volume fraction $f_{2}$ of the second phase by adding an extra layer of matrix material becomes

$$
d f_{2}=-f_{2} \frac{3 l^{2} d l+3 l d l^{2}+d l^{3}}{l^{3}+3 l^{2} d l+3 l d l^{2}+d l^{3}},
$$

or omitting the high-order terms

$$
d f_{2}=-3 f_{2} \frac{d l}{l},
$$

which in combination with Eq. (5) leads to

$$
\frac{3 E_{1}}{\left(E^{2}+E E_{1}-2 E_{1}^{2}\right)} d E=\frac{1}{f_{2}} d f_{2} .
$$

Performing the integration of Eq. (8) from the $f_{2}=1$ with $E=E_{2}$ to $f_{2}=f_{2}$ with $E=E$, the $E$ modulus of the cubic composite is finally derived:

$$
E_{\text {cube }}=E_{1} \frac{3 E_{2}+2 f_{1}\left(E_{1}-E_{2}\right)}{3 E_{1}+f_{1}\left(E_{2}-E_{1}\right)} .
$$

As this value is obtained both as lowest upper bound [of all $(\mathrm{PS})^{n}$ ] and highest lower bound [of all (SP) ${ }^{n}$ ], it is suggested to be the best approximation for the actual value of the $E$ modulus of a composite of this type, i.e., which can be represented by a cube of a second phase in a matrix cube.

\section{GENERALIZATIONS}

In an analogous way, the existence of a converging $E$ modulus can be proven for the case with a continuous secondary phase of fibers with a quadratic cross section surrounded by a square matrix [this case can be gained from Fig. 2(a), by allowing one of the horizontal edge lengths of the second phase becoming equal to that of the unit cell of the composite]:

$$
E_{\text {square }}=E_{1} \frac{2 E_{2}+f_{1}\left(E_{1}-E_{2}\right)}{2 E_{1}+f_{1}\left(E_{2}-E_{1}\right)} .
$$

Equations (9) and (10) are the final expressions for Young's modulus of composites of 3D embedding type (represented by a cube in cube) and an effectively $2 \mathrm{D}$ penetrating type (represented by a square cross section in square), in which $E_{1}, f_{1}$ and $E_{2}, f_{2}$ are the elastic moduli and volume fractions of the matrix and the second phase, respectively.

In the above treatment, a regularly distributed second phase of cubic shape has been considered. As inclusions in actual cases are neither cube shaped nor equiaxed nor perfectly regularly distributed with same spacing in three directions, a more general case is outlined, where a cuboid of a second phase is embedded in a matrix cuboid with arbitrary edge lengths, as illustrated in Fig. 2(b). In order 
TABLE I. Several particular cases for the arrangement of a second phase in the matrix of two-phase composites: their geometrical conditions to derive them from the general unit cell of Fig. 2(b), their corresponding shape/distribution parameter $\beta$ for use with the general equation, Eq. (12), for cuboid two-phase composites, and the simplified equation obtained in this manner.

\begin{tabular}{lccc}
\hline \hline Arrangement & $f(A, B, C, a, b, c)$ & $\beta$ & Eqs. \\
\hline Series connection & $A=a$ and $B=b$ & 1 & $(1)$ \\
Parallel connection & $C=c$ & 0 & $(2)$ \\
3D cube in cube & $A=B=C$ and $a=b=c$ & $1 / 3$ & $(9)$ \\
2D square in square & $A=C$ and $a=c$ and $B=b$ & $1 / 2$ & $(10)$ \\
\hline \hline
\end{tabular}

to describe the different nesting shells and to perform the integration of the matrix volume, the increments in the different principal directions of the aligned cuboids are linked to each other by $d a /(A-a)=d b /(B-b)=d c /(C-c)$. Following analogous procedures, the convergence of the upper and lower bounds of the increment $d E$ calculated by SP and PS connections can be proven. Omitting the high-order terms, the differential expression becomes

$$
\begin{aligned}
d E_{P S} & =d E_{S P} \\
& =\frac{\left(E_{1}^{2}-E E_{1}\right) d t_{1}+\left(E_{1}^{2}-E E_{1}\right) d t_{2}+\left(E^{2}-E E_{1}\right) d t_{3}}{E_{1}},
\end{aligned}
$$

where $d t_{1}=d a / l_{a}, d t_{2}=d b / l_{b}$, and $d t_{3}=d c / l_{c}$. Performing the integration and substituting the upper and lower limits, one obtains as best approximation for the Young's modulus of the cuboid composite:

$$
\begin{aligned}
& E_{\text {cuboid }}=E_{1} \frac{E_{2}\left(f_{2}+\beta f_{1}\right)+E_{1}\left(f_{1}-\beta f_{1}\right)}{E_{2} \beta f_{1}+E_{1}\left(1-\beta f_{1}\right)} \\
& \text { with } \frac{1}{\beta}=1+\frac{C(B-b)}{B(C-c)}+\frac{C(A-a)}{A(C-c)},
\end{aligned}
$$

in which $E_{1}, f_{1}$ and $E_{2}, f_{2}$ are the elastic moduli and volume fractions of the matrix and second phase, respectively. The parameter $\beta$ in Eq. (12) reflects the effect of the shape and the distribution of the second phase on the composite modulus, hence, $\beta$ is termed shape/distribution parameter.

From the general expression in Eq. (12), several particular cases can be derived by applying certain geometrical restrictions on the different side lengths of the cuboids. Table I lists the obtainable cases for the different arrangements, the geometrical conditions, the resulting shape/distribution parameter $\beta$ as well as the simplified equations obtained for the specific cases. Consequently, Eq. (12) can be seen as generalized approximation for the Young's modulus of two-phase materials formed by discrete inclusions of arbitrary aspect ratios regularly distributed in the matrix.

The generalized treatment for the Young's modulus can be straightforwardly extended to the shear modulus $G$ as well, because the expressions for the shear modulus of a composite are the same as that of the $E$ modulus in both extreme cases, i.e., for both the series and the parallel connection [26]. Following the same derivation process, the final approximations for $G$ for the considered two-phase composites are completely analogous to those for $E$, i.e.,

$$
\begin{gathered}
G_{\text {cuboid }}=G_{1} \frac{G_{2}\left(f_{2}+\beta f_{1}\right)+G_{1}\left(f_{1}-\beta f_{1}\right)}{G_{2} \beta f_{1}+G_{1}\left(1-\beta f_{1}\right)} \\
\text { with } \frac{1}{\beta}=1+\frac{C(B-b)}{B(C-c)}+\frac{C(A-a)}{A(C-c)}, \\
G_{\text {cube }}=G_{1} \frac{3 G_{2}+2 f_{1}\left(G_{1}-G_{2}\right)}{3 G_{1}+f_{1}\left(G_{2}-G_{1}\right)}, \\
G_{\text {square }}=G_{1} \frac{2 G_{2}+f_{1}\left(G_{1}-G_{2}\right)}{2 G_{1}+f_{1}\left(G_{2}-G_{1}\right)},
\end{gathered}
$$

For elastically anisotropic composites, Eqs. (12) and (13) should be also adequate for estimating the Young's and shear moduli along specific loading directions as long as one knows the Young's and shear moduli of the two constituent phases along the corresponding directions. If the two-phase composite is elastically isotropic, Poisson's ratio can be further calculated from the relation for isotropic media:

$$
v_{\text {composite }}=\frac{E_{\text {composite }}}{2 G_{\text {comosite }}}-1,
$$

in which the values of $E_{\text {composite }}$ and $G_{\text {composite }}$ represent the ones calculated by Eqs. (12) and (13).

\section{VERIFICATION BY FEM SIMULATIONS}

To proof the validity and usefulness of the obtained approximation for the Young's modulus, FEM simulations of the corresponding idealized unit cell have been performed. Figure 3 shows the strain distributions of the cubic unit cells of two-phase composites with different volume fractions $f_{2}=0.05$ and 0.5 of the cubes of second phase under purely elastic loading. As an example, the behavior is simulated for matrices that are more compliant or stiffer than the inclusion, i.e., the Young's modulus of the matrix is smaller ("compliant matrix") or higher ("stiffer matrix") than the Young's modulus of the inclusion.

From Fig. 3, it can be seen that the strain distribution is rather heterogeneous and reveals the obvious feature that the strain changes gradually from inside to outside like an onion structure analogously to the above suggested integration process. Comparison of Figs. 3(a) and 3(c) with Figs. 3(b) and $3(\mathrm{~d})$ illustrates that the more compliant phase always experiences larger elastic strains than the stiffer one. Hence larger elastic moduli can be expected for the composites with stiff matrices than for the one with compliant matrices for the same volume fraction at least in the investigated range of volume fractions in accordance with Eq. (9). These features indicate a close correspondence between the above derivation of approximate expressions and the simulated behavior.

Figure 4 presents a quantitative comparison between the approximate values (represented by lines) predicted by the above equations and the numerical results for the macroscopic behavior of the FEM simulations. For the 2D interpenetrating type (square in square) and the 3D embedding type (cube in cube), the FEM simulation results (marked by dots) shown in Figs. 4(a) and 4(b) are nicely consistent with the analytic 


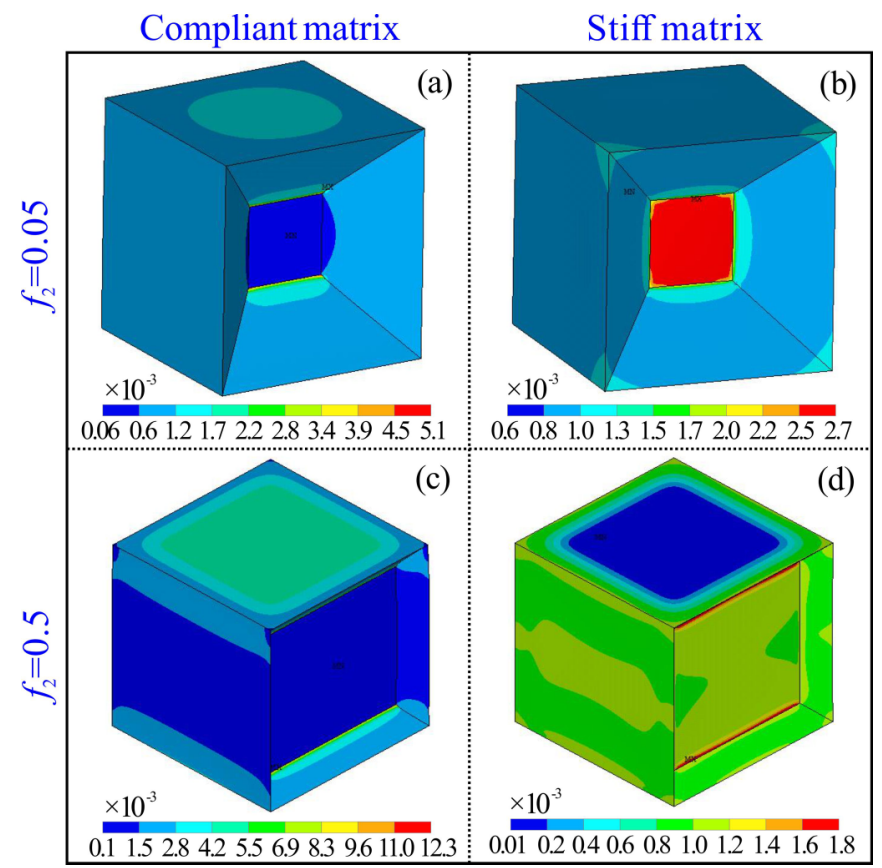

FIG. 3. Strain distributions obtained by FEM for composites with cubic unit cells of matrix material containing a cubic inclusion for different volume fractions of the second phase $\left(f_{2}=0.05\right.$ or 0.5$)$ under pure elastic loading. For an easier viewing of the strains in the unit cell, part of it has been removed on the face facing right. Two different cases are illustrated: in the left column the matrix is more compliant than the inclusion (compliant matrix) and in the right column the matrix is stiffer than the inclusion (stiff matrix).
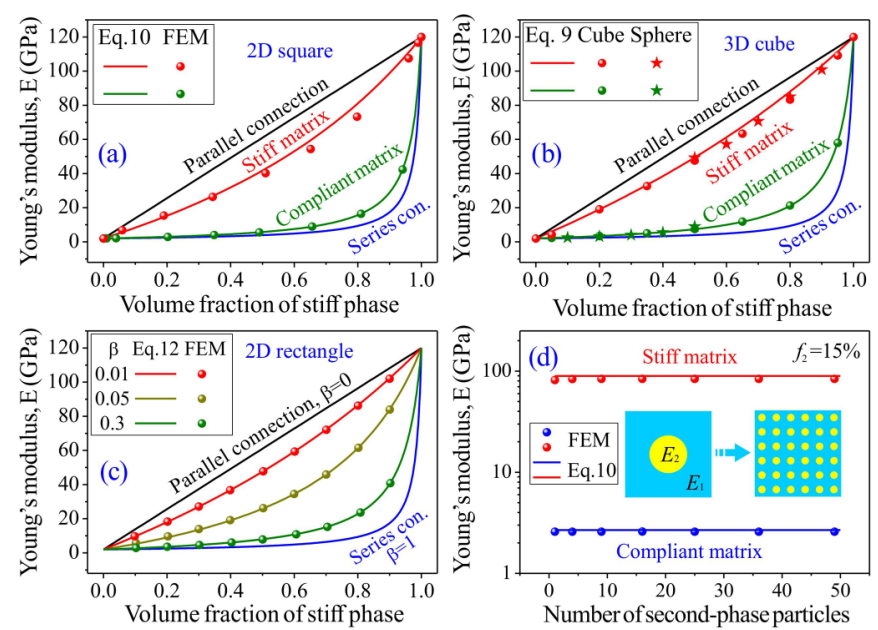

FIG. 4. Comparison between the proposed analytical model and the results of FEM simulations for different particular arrangements of unit cell of two-phase composites: (a) 2D interpenetrating case (square cross section in square), (b) 3D embedding case (cube in cube); a FEM simulation of a spherical inclusion in a matrix cube is also included, (c) rectangular cross section in rectangle and (d) circular cross section(s) in square matrix. The results in (a)-(c) confirm the adequacy of the derived analytical expressions [Eqs. (9), (10), and (12)]; (d) proves the rationality of the consideration that a basic unit cell can represent the elastic properties of a composite with regularly (periodically) arranged inclusions. predictions for both, the "stiff matrix" case and the "compliant matrix" case.

Considering that in real composites the embedded second phase particles are often spherical, additional FEM simulations of spherical particles in a 3D matrix cube were performed by replacing the cube of second phases with a sphere. The corresponding results are marked by stars in Fig. 4(b); they also exhibit a fairly good accordance with the analytical prediction for cubes in a matrix cube (Note that the largest volume fraction of a sphere in a cube is 0.52 ). These results indicate that the detailed morphology of an equiaxed particle of a second phase has only minor influence on the modulus of the composite, which will be further evidenced later by the experimental data. On the other hand, the modulus of the composite will be largely affected by the aspect ratio and arrangement of the particles of the second phase, as quantified in Eq. (12) through the shape/distribution parameter $\beta$. Figure 4(c) shows this effect for the $2 \mathrm{D}$ interpenetrating case of a rectangular cross section in a matrix rectangle via varying the edge length of both, the rectangular second phase and matrix, through varrying the value of the parameter $\beta$. The FEM simulation results in Fig. 4(c) are consistent with the prediction of the analytical expressions for all different $\beta$ in the investigated range. This clearly indicates that the present model is capable of predicting the elastic properties of composites with not only equiaxed second phase particles, but also particles with quite anisotropic shapes and/or arrangements. Finally, Fig. 4(d) presents FEM results of the Young's modulus of a square matrix containing circular particles of different size and number $\left(1,2^{2}, 3^{2}, 4^{2}, 5^{2}, 6^{2}\right.$, and $\left.7^{2}\right)$ arranged in a square grid, but amounting to the same total volume fraction $(15 \%)$. It shows that the modulus of the corresponding composites not only has the same value as predicted by the model; it also becomes independent of the size and the number of the particles for both, stiff and compliant matrices. This confirms the rationality of the consideration above, that a basic unit cell can represent the elastic properties of the composite with regularly arranged particles, i.e., that elastic interactions between particles do not have to be considered explicitly in the unit cell model when calculating the elastic moduli of the composites, which is the basic assumption for the application of the present model.

\section{VERIFICATION BY THE PREVIOUS EXPERIMENTAL DATA}

Figure 5 shows a comparison between plenty of experimental results from literature [27-38] and the analytical predictions of our model for the Young's modulus (a)-(g), the shear modulus (h), and Poisson's ratio (i). Upper and lower bounds from several classical model are additionally provided in Fig. 5 for comparison purposes: Voigt and Reuss bounds (VR bounds) [2], Hashin and Shtrikman bounds (HS bounds) [14] and Ravichandra bounds (R bounds) [17]. All of these experimental studies used a similar method for preparing the two-phase composite by mixing two phases: equiaxed (spherical) hard second particles or phases were added homogeneously into the soft matrixes. The analytical approximations derived in this study in Eqs. (9), (13), and (16) were tested against these different composites. It can be 


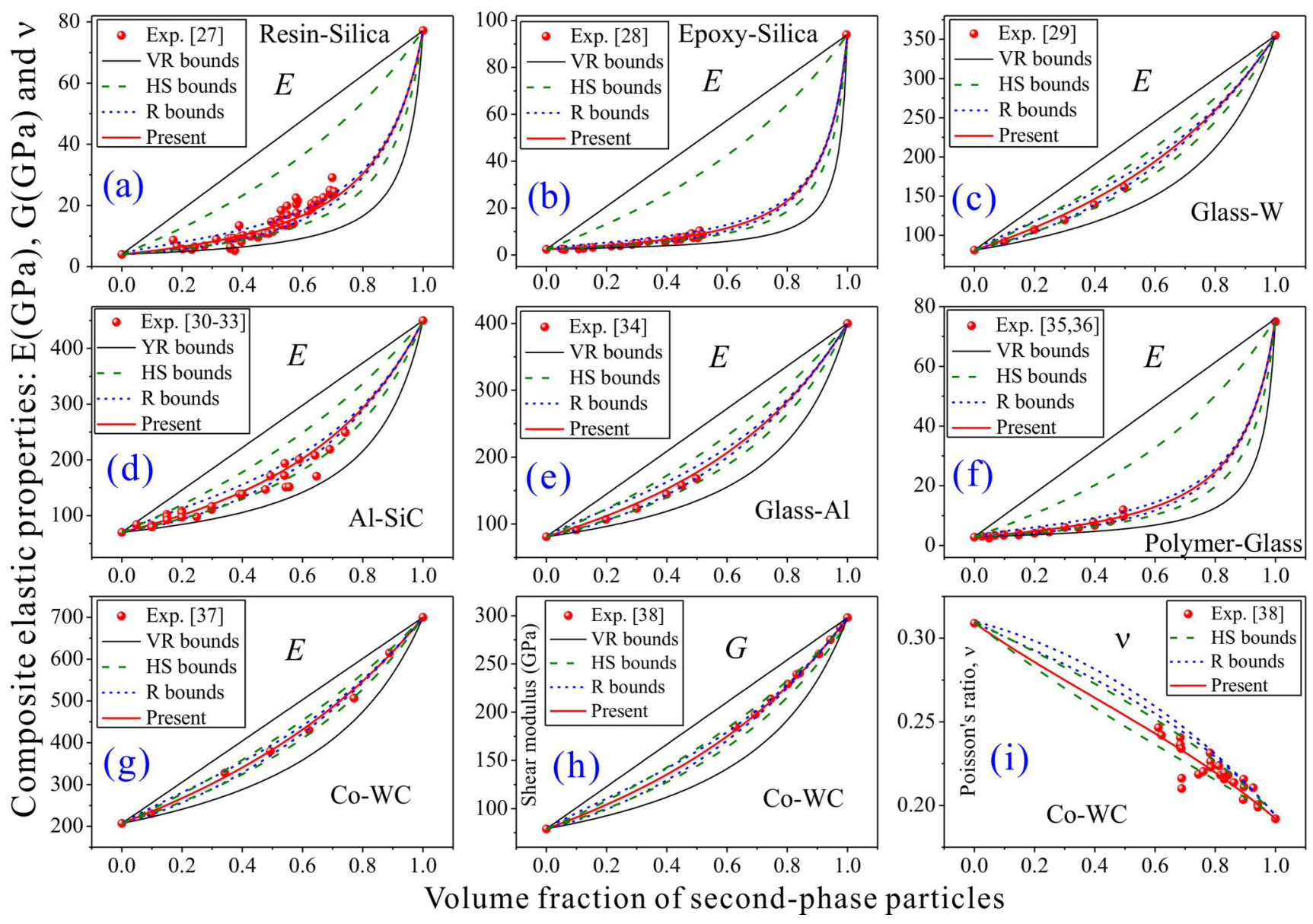

FIG. 5. Comparisons between several model predictions and the analytical expressions derived here with reported experimental results for elastic properties of two-phase composites: (a)-(g) Young's modulus, (h) shear modulus, and (i) Poisson's ratio. It shows that the suggested model leads to more concise and accurate predictions than previous bounds.

seen that in all cases, the predicted values from the analytical expressions lie in the middle of the three sets of bounds as expected, and that, most importantly and unlike these bounds, the analytical approximations essentially describe all experimental data for various metallic and nonmetallic composites neatly. Consequently, the presented equations give a more concise and accurate prediction than the previous bounds. These results combined with the above FEM simulations reveal that the integration method proposed in this study for obtaining an analytical approximation for the elastic constants of isotropic two-phase composites presents a practical and reasonable approach.

\section{ADVANTAGES AND APPLICABILITY OF THE MODEL}

Relative to previous models and methods for predicting the elastic properties of two-phase composites, the present approach has three major advantages: (1) the derivation is briefer and the final analytical expressions are (i) simpler in form and (ii) provide a more accurate prediction. Although similar arrangements of connection sequences have been used in previous attempts, the number of dismounting and joining steps have been limited to two or four due to tedious iterative computations and only upper and lower limiting bounds could be acquired $[17,18]$. In the present model, through modifying the representative units, the number of dismounting and joining steps were increased to infinity so that an integral method could be introduced in the derivation, which allows the derivation of well-defined approximate values for the elastic moduli. (2) The present model is handier in predicting the Young's modulus and the shear modulus of two-phase composite, because solely the Young's and shear moduli of the constituents are required without the need for knowledge of other elastic constants, as indicated by Eqs. (12), (13), and (16). (3) Most importantly, the proposed analytical approximations have a more wide applicability even on cases not explicitly considered in their derivation. Basically, the above mentioned bounds $[17,18]$ can only deal with composites with equiaxed and regularly distributed second phases. Nevertheless, as supported by the FEM results [Fig. 4(c)], the unified equations of the present model are also suitable for two-phase composites that even have nonequiaxed second phases in anisotropic arrangements, as reflected by the proposed shape/distribution parameter $\beta$.

Aside from the elastic moduli, other physical properties of the composite that possess linear constitutive relations may also be tackled by the same method. For example, the governing relations for the electrical conductivity, i.e., the relationship between voltage and electrical current density, are completely analogous to those for the Young's modulus and its relation on stress and strain (at least in the isotropic 
case). Therefore the derivation and the final expressions for the effective conductivity of the composite should resemble the results derived above.

\section{CONCLUSION}

In the present study, an integration method is proposed to derive analytical approximations, based on the equations of series and parallel connections, for the elastic constants of two-phase materials. Explicit expressions were derived for the Young's modulus, the shear modulus and (in case of elastic isotropy) the Poisson's ratio of two-phase composites consisting of a second phase dispersed in a matrix for various volume fractions, shapes and arrangements of the second phase. Finally, the various FEM simulations performed and numerous experimental data consulted verified the adequacy and general applicability of the obtained analytical expressions.

\section{ACKNOWLEDGEMENTS}

This work was financially supported by the National Natural Science Foundation of China (NSFC) under grant Nos. 51401207, 51331007, and 51201165.
[1] D. R. Clarke, J. Am. Ceram. Soc. 75, 739 (1992).

[2] K. K. Chawla, Composite Materials: Science and Engineering (Springer Science \& Business Media, 2012).

[3] R. Everett and R. J. Arsenault, Metal Matrix Composites: Mechanisms and Properties (Academic Press, New York, 1991).

[4] D. S. McLachlan, M. Blaszkiewicz, and R. E. Newnham, J. Am. Ceram. Soc. 73, 2187 (1990).

[5] M. A. Meyers, P.-Y. Chen, A. Y.-M. Lin, and Y. Seki, Prog. Mater. Sci. 53, 1 (2008).

[6] J. L. Katz, J. Biomech. 4, 455 (1971).

[7] G. P. Sendeckyj, J. Compos. Mater. 3, 177 (1969).

[8] R. H. Krock, in Modern Developments in Powder Metallurgy: Volume 3 Development and Future Prospects (Springer US, Boston, MA, 1966), p. 105.

[9] J. P. Watt, G. F. Davies, and R. J. O'Connell, Rev. Geophys. 14, 541 (1976).

[10] J. G. Wang, C. F. Leung, and Y. Ichikawa, Comput. Geotech. 29, 477 (2002).

[11] J. R. Willis, Mech. Solids The Rodney Hill 60th Anniversary Volume (1982), http://www.sciencedirect.com/science/article/ pii/B9780080254432500252.

[12] B. Budiansky, J. Mech. Phys. Solids 13, 223 (1965).

[13] T. T. Wu, Int. J. Solids Struct. 2, 1 (1966).

[14] Z. Hashin and S. Shtrikman, J. Mech. Phys. Solids 11, 127 (1963).

[15] R. Hill and J. Mech. Phys. Solids 11, 357 (1963).

[16] B. Paul, Prediction of Elastic Constants of Multiphase Material. Technical Report No. 3, Brown University, 1959.

[17] K. S. Ravichandran, J. Am. Ceram. Soc. 77, 1178 (1994).

[18] G. Bourkas, I. Prassianakis, V. Kytopoulos, E. Sideridis, and C. Younis, Adv. Mater. Sci. Eng. 2010, 891824 (2010).

[19] E. D. Yilmaz, H. Jelitto, and G. A. Schneider, Acta Biomater. 16, 187 (2015).
[20] T. T. Wu, J. Appl. Mech. 32, 211 (1965).

[21] L. P. Liu, Proc. R. Soc. A 466, 3693 (2010).

[22] A. P. Roberts and E. J. Garboczi, Acta Mater. 49, 189 (2000).

[23] E. J. Garboczi and J. G. Berryman, Mech. Mater. 33, 455 (2001).

[24] S. Meille and E. J. Garboczi, Mod. Sim. Mater. Sci. Eng. 9, 371 (2001).

[25] A. P. Roberts and E. J. Garboczi, Proc. Royal Soc. 458, 1033 (2002).

[26] T. G. Richard, J. Compos. Mater. 9, 108 (1975).

[27] M. Braem, V. E. Van Doren, P. Lambrechts, and G. Vanherle, J. Mater. Sci. 22, 2037 (1987).

[28] O. Ishai and L. J. Cohen, Int. J. Mech. Sci. 9, 539 (1967).

[29] D. P. H. Hasselman and R. M. Fulrath, J. Am. Ceram. Soc. 48, 548 (1965).

[30] I. A. Ibrahim, F. A. Mohamed, and E. J. Lavernia, J. Mater. Sci. 26, 1137 (1991).

[31] V. K. Lindroos and M. J. Talvitie, J. Mater. Process. Tech. 53, 273 (1995).

[32] A. Mortensen, in Proceedings of International Conference on Fabrication of Particulates Reinforced Metal Composites, 1990, pp. 217-233.

[33] M. K. Premkumar, W. H. Hunt, and R. R. Sawtell, JOM 44, 24 (1992).

[34] D. P. H. Hasselman and R. M. Fulrath, J. Am. Ceram. Soc. 48, 218 (1965).

[35] J. C. Smith, Polym. Eng. Sci. 16, 394 (1976).

[36] J. C. Smith, J. Res. NBS 80A, 45 (1976).

[37] M. Nishimatsu and J. Gurland, Trans. ASM 52, 469 (1960).

[38] H. Doi, Y. Fujiwara, K. Miyake, and Y. Oosawa, Metall. Mater. Trans. 1, 1417 (1970). 